\title{
Comparison of the rates for reaching the blastocyst stage between normal and abnormal pronucleus embryos monitored by a time-lapse system in IVF patients
}

\author{
(D) Kübra Nur Uzun 1 , (D) Mehmet Cincık², (D) Belgin Selam³, (D) Özgüç Takmaz³, (D) Esra Uyar4 \\ ${ }_{1}^{1} \mathrm{PhD}$ Student, Institute of Health Sciences, İstanbul Medipol University, İstanbul, Turkey \\ 2Department of Histology and Embryology, Maltepe University Faculty of Medicine, İstanbul, Turkey \\ 3Department of Obstetrics and Gynecology, Acıbadem Mehmet Ali Aydınlar University Faculty of Medicine, İstanbul, Turkey \\ 4Unit of ART, Acıbadem Altunizade Hospital, İstanbul, Turkey
}

\section{Abstract}

Objective: To compare the rates of blastocyst stage development between embryos fertilized after one (MPN) or more than two pronucleus (PN) (3PN, 4PN-multiPN) with those after 2PN in the same patients.

Material and Methods: The embryos of patients who had both abnormal PN (MPN, 3PN or 4PN) and normal fertilized (2PN) embryos after intracytoplasmic sperm injection (ICSI) fertilization, were followed with a time-lapse system following the ICSI procedure. The rates of reaching the blastocyst stage were compared between normal and abnormally fertilized embryos.

Results: One thousand eight hundred and twenty oocytes were collected from 140 patients and 1280 (70.3\%) of them were fertilized. MPN, 2PN and 3PN, 4PN (multiPN) ratios of the embryos in the pronuclear stage were $11.4 \%, 83.13 \%$ and $5.47 \%$, respectively. The rates of reaching the blastocyst stage among these embryos were $17.1 \%, 60.8 \%$ and $42.8 \%$ for MPN, $2 \mathrm{PN}$ and multiPN, respectively. The proportion reaching blastocyst development was significantly higher following $2 \mathrm{PN}$ compared to those after MPN and multiPN ( $<<0.05)$. Embryos developing after multiPN had significantly higher rates of reaching the blastocyst stage compared to those after MPN $(\mathrm{p}<0.01)$.

Conclusion: The majority of abnormally pronucleated embryos arrest without reaching the blastocyst stage. MultiPN embryos have a higher rate of blastocyst development than MPN embryos. (J Turk Ger Gynecol Assoc 2021; 22: 120-6)

Keywords: Blastocyst, ICSI, MPN, multiPN, time-lapse

Received: 12 March, 2020 Accepted: 20 July, 2020

\section{Introduction}

Successful fertilization is the first parameter that is checked following the combination of oocyte and sperm in assisted reproduction treatments. Two pronucleus $(\mathrm{PN})$ and two polar bodies (PB) are observed 16-18 hours after fertilization under normal conditions. However, an abnormal number of pronuclei $(1,3$ or 4$)$ can be seen if abnormal fertilization has occurred.
The reason for the presence of only one pronucleus (MPN) in the embryo after fertilization has been investigated by many researchers (1-10). MPN and one or two PB's are observed in $2.7-17 \%$ of embryos after in vitro fertilization (IVF) and intracytoplasmic sperm injection (ICSI) procedures (1). Embryos with MPN usually have two PB's (1). Plachot (2) reported that the incidence of MPN was around 1\% in IVF or ICSI cycles.

The zygotes with MPN were thought to be due to parthenogenetic activation rather than spermatozoon fertilization (3). However, 
sperm penetration findings were observed in approximately $45 \%$ of MPN zygotes, providing evidence for their development after fertilization (4). The presence of $\mathrm{Y}$ chromosomes in the genetic structure of MPN zygotes with preimplantation genetic diagnosis was reported as a proof of fertilization (5). Most embryos that develop from MPN have been shown to be aneuploid, even though they contain Y chromosomes (6).

The presence of MPN may be due to errors in the formation or fusion of the pronucleus during fertilization (7). Failure of male or female chromatids to form a pronucleus causes MPN. Flaherty et al. (8) observed swollen sperm head (52\%), intact, undecondensed sperm head (28\%), and ejection of the spermatozoon (20\%) in one-pronuclear oocytes. The formation of MPN embryos was also based on the asynchronous occurrence of pronucleus, the union of male and female pronucleus, and male or female parthenogenesis (9). The asynchronous view of pronucleus formation has been assessed as the most likely explanation for the presence of biparental diplodia in embryos developing from MPN and some of these embryos were reported to be transferable (10).

The presence of more than two pronuclei in the fertilization control stage, rather than the expected $2 \mathrm{PN}$, is called multiPN. The rate of $3 \mathrm{PN}$ formation was $1 \%$ and $5 \%$ after ICSI and IVF procedures, respectively (11). Feenan and Herbert (12) considered the presence of more than two pronuclei to be associated with genetic disorders. They concluded that out of 3PN embryos, $61.8 \%$ had a triploid chromosome component, $25.2 \%$ had mosaic sequencing, and only $12.6 \%$ carried a diploid chromosome set (12). More than two PN formation may be triggered by inability to extrude the second polar body, incomplete chromatid separation to the extruded polar body, and dispersion of oocyte chromatids in the second polar body formation (13).

Early cleavage may appear normal in 3PN zygotes, but progression may cease or aneuploidy may occur later (14). After IVF, 3PN zygotes may develop into different embryo stages with variable chromosomal component (triploidy; XXY, XXX, XYY, diploidy, mosaic) (15).

A time-lapse system allows observation of embryonic development in detail and provides algorithms to select high quality embryos according to the kinetic and morphologic changes. It is important to select embryos with high implantation potential for transfer in assisted reproduction. Abnormally fertilized oocytes, depending on how they are formed, either develop to different stages or arrest, usually from the first day. Those which develop to the fifth day are not preferred for transfer because they have low scores. The aim of this study was to compare the rates of reaching the blastocyst stage among abnormally fertilized embryos developing after
MPN and multiPN with those of the normally fertilized embryos after 2PN using a time-lapse system.

\section{Material and Methods}

\section{Study design}

This was a retrospective study conducted on IVF patients treated at an IVF center, between January 2013 and December 2017. The study protocol was approved by the Institutional Review Board of the Maltepe University Faculty of Medicine (approval number: 78, date: 22.09.2017). The study included 140 patients. Consent forms were obtained from the patients. Inclusion criteria were patients aged 25-40 years, patients who had both abnormal PN (MPN, 3PN or 4PN) and normal fertilized (2PN) embryos after fertilization, with all embryos followed with a time-lapse system following an ICSI procedure. The development of fertilized oocytes from the first day to the fifth day was examined. The rates of reaching the blastocyst stage between normal and abnormally fertilized embryos were compared.

\section{Ovarian stimulation}

The standard ovarian stimulation protocol of our clinic has been reported previously (16). Pituitary down-regulation was performed either by the gonadotropin-releasing hormone agonist (GnRHa) leuprolide acetate (lucrin: $0.5 \mathrm{mg} / \mathrm{mL}$, Abbott, Spain) or GnRH antagonist, cetrorelix acetate (Cetrotide, Baxter Oncology $\mathrm{GmbH}$, Germany). Leuprolide acetate was used daily in the late luteal phase before the treatment cycle and cetrorelix acetate was started daily on the $5^{\text {th }}$ day of the treatment and was continued until ovulation was triggered. Gonadotropin injections were started on cycle days 2 or 3 , if $>2 \mathrm{~cm}$ cysts were not observed by baseline ultrasound in patients. The daily dosage for gonadotropin stimulation was individualized between 150 and 300 IU. Patients were monitored by ultrasound until the trigger criteria, including three follicles with maximum diameter $>17 \mathrm{~mm}$, were present. Human chorionic gonadotropin (hCG) $10000 \mathrm{U}$ (Choriomon, IBSA, Italy) and $5000 \mathrm{U}$ hCG (Choriomon, IBSA, Italy) plus $0.2 \mathrm{mg}$ triptorelin acetate (Gonapeptyl, Ferring $\mathrm{GmbH}$ Liel, Germany) were used for oocyte maturation in the agonist cycles and the antagonist cycles, respectively. Patients underwent transvaginal ultrasound-guided oocyte retrieval with a 17-gauge needle, under general anesthesia, 35-36 hours after hCG administration. The oocyte-corona complexes were denuded, incubated for 2 hours and ICSI was performed. ICSI is routinely performed in all patients as our clinical policy.

\section{Preparing EmbryoScope dish}

Micro-wells of the EmbryoScope dish were filled with prewarmed culture solution. Wells were filled with $\sim 25 \mu$ l solution without any bubbles and were covered with $1.4 \mathrm{~mL}$ ovoil. The 
dishes were incubated at $37{ }^{\circ} \mathrm{C}$. Each injected oocyte was placed in a well without any bubbles and slides were placed into the time-lapse system (EmbryoScope, Vitrolife, Sweden).

\section{Embryo selection}

Patient information and the quality of embryos observed were recorded every day by the EmbryoScope. The main issue for selection of an embryo for transfer was to be at an appropriate stage according to post-insemination time. Size and number of PNs were considered. Size and evenness of the blastomere, fragmentation ( $>10 \%)$, multinucleation, vacuole $(>14 \mu \mathrm{m})$, and cleavage times were evaluated at the early cleavage stages. Cell numbers that join the cell compaction were considered for the next stage, and then, inner cell mass and trophectoderm were notated for the blastocyst stage embryo.

\section{Statistical analysis}

All analyses were carried out by Number Cruncher Statistical System 2007 (Kaysville, Utah, USA). Univariate and mulivariate Generalized Linear Mixed models were used to examine the effect of PN number on blastocyst development and the effect of age and etiologies of infertility on PN number. A p $<0.05$ was considered to indicate statistical significance.

\section{Results}

Demographic and clinical characteristics of the patients are summarized in Table 1.

\section{Table 1. Demographic and clinical characteristics of} the patients

\begin{tabular}{|c|c|c|}
\hline \multicolumn{3}{|l|}{$(n=146)$} \\
\hline \multirow{2}{*}{ Age (years) } & Range & $25-40$ \\
\hline & Mean and standard deviation & $32.74 \pm 4.32$ \\
\hline \multirow{2}{*}{ BMI } & Range & $16.2-41.5$ \\
\hline & Mean and standard deviation & $23.48 \pm 4.48$ \\
\hline \multicolumn{2}{|l|}{ Indications } & n (\%) \\
\hline & Unexplained & $46(31.5)$ \\
\hline & Male & $46(31.5)$ \\
\hline & Anovulation & $14(9.6)$ \\
\hline & Tubal factor & $23(15.8)$ \\
\hline & Endometriosis & $15(10.3)$ \\
\hline & Poor ovarian reserve & $16(11.0)$ \\
\hline \multirow{5}{*}{$\begin{array}{l}\text { Number of } \\
\text { previous embryo } \\
\text { transfer }\end{array}$} & 0 & $37(25.2)$ \\
\hline & 1 & $9(6.2)$ \\
\hline & 2 & $96(65.8)$ \\
\hline & 3 & $2(1.4)$ \\
\hline & 4 & $2(1.4)$ \\
\hline
\end{tabular}

The number of oocytes collected from the 140 patients in the study was 1820 . Fertilization was observed in $70.3 \%(n=1280)$ of the oocytes (Table 2). Among the fertilized oocytes, 146 were MPN (11.4\%), 1064 were 2PN (83.1\%) and 70 were multiPN (5.5\%) (Table 2). Abnormal fertilization (MPN, multiPN) was observed in 216 embryos with an abnormal fertilization rate of $16.9 \%$. Out of 1,280 fertilized embryos, 702 (54.8\%) reached the blastocyst stage.

The embryos were divided into three groups, MPN, 2PN and multiPN, according to the number of pronuclei observed on the first day and the rates of blastocyst development were calculated for each group. Blastocyst development was observed in $17.1 \%, 60.8 \%$ and $42.9 \%$ of the embryos developing after MPN, 2PN and multiPN, respectively (Table 3).

A generalized linear mixed models analysis was used to examine the effect of PN number on blastocyst development. The model obtained was statistically significant, thus the number of PN had a statistically significant effect on the success of blastocyst development $(\mathrm{F}=43,731 ; \mathrm{p}<0.001)$ (Table 4). When binary evaluations were examined, blastocyst development percentage was higher in the $2 \mathrm{PN}$ oocytes than MPN and multiPN groups ( $\mathrm{p}<0.001 ; \mathrm{p}=0.015$, respectively). When abnormally fertilized groups were analyzed, blastocyst development rate in the multiPN group was significantly higher than in the MPN group $(\mathrm{p}<0.001)$. When the association between abnormal PN incidence and patients' characteristics was analyzed, no significant differences were found in the MPN/multiPN incidence when donor women were divided into age groups $<35$ years and $\geq 35$ years of age $(p>0.05)$ (Table 5).

The rates of abnormal PN between groups stratified by etiology of infertility was also investigated. No significant difference was found between the rates of multiPN among the infertility etiology groups by post-hoc analysis ( $p>0.05)$. The rate of MPN in the poor ovarian reserve group was significantly higher than those of the other etiological groups $(\mathrm{p}<0.05)$.

Table 2. Fertilization and blastocyst development among the study group

\begin{tabular}{|l|l|l|l|}
\hline \multirow{2}{|c|}{} & n & $\%$ \\
\hline \multirow{3}{*}{ Fertilization } & No & 540 & 29.7 \\
\cline { 2 - 4 } & Yes & 1280 & 70.3 \\
\hline \multirow{3}{*}{ PN (n=1280) } & MPN & 146 & 11.4 \\
\cline { 2 - 5 } & $2 \mathrm{PN}$ & 1064 & 83.1 \\
\cline { 2 - 5 } & MultiPN & 70 & 5.5 \\
\hline \multirow{2}{*}{ Blastocyst development (n=1280) } & No & 578 & 45.2 \\
\cline { 2 - 5 } & Yes & 702 & 54.8 \\
\hline PN: Pronucleus number, MPN: Multiple pronuclear & & \\
\hline
\end{tabular}




\section{Discussion}

The current study demonstrated that most of the embryos developing after MPN and multiPN did not reach the blastocyst stage. The proportion of those that reached the blastocyst stage was higher in multiPN embryos than in MPN embryos. The development of blastocyst from embryos with two pronuclei was significantly higher when compared to the other two groups with abnormal fertilization. According to this data, selection of embryos with an abnormal number of pronuclei for transfer is not recommended in routine practice. However, a small number of embryos that fertilized with an abnormal number of pronuclei in the oocyte can reach the blastocyst stage in some patients. Transfer of these embryos should be considered carefully.

This was a retrospective study and we evaluated patients having both normal and abnormally fertilized embryos. As patients already had 2PN embryos, further tests including trophoectoderm biopsy and PGS were not performed for abnormal PN embryos which reached the blastocyst stage to check the possibility of self-correction. In addition, blastoscysts

Table 3. Development of blastocyst according to pronucleus number

\begin{tabular}{|l|l|l|l|}
\hline \multicolumn{2}{|c|}{} & Total & $\begin{array}{l}\text { Development of } \\
\text { blastocyte }\end{array}$ \\
\cline { 3 - 4 } \multicolumn{2}{|c|}{} & n & n (\%) \\
\hline \multirow{3}{*}{ PN } & MPN & 146 & $25(17.1)$ \\
\cline { 2 - 4 } & 2PN & 1064 & $647(60.8)$ \\
\cline { 2 - 4 } & MultiPN & 70 & $30(42.9)$ \\
\hline \multicolumn{2}{|l|}{ PN: Pronucleus number } \\
\hline
\end{tabular}

Table 4. Generalized Linear Mixed Models for the effect of PN number on blastocyst development

\begin{tabular}{|l|l|l|l|}
\hline \multirow{2}{*}{} & \multicolumn{3}{|l|}{ Model } \\
\cline { 2 - 4 } & Beta & Exp (beta) $\mathbf{( 9 5 \%}$ CI) & p \\
\hline PN (MPN) & - & 1 & - \\
\hline PN (2PN) & 2,148 & $8,570(5,405 ; 13,588)$ & $<\mathbf{0 . 0 0 1} 1^{* *}$ \\
\hline PN (multiPN) & 1,504 & $4,501(2,294 ; 8,828)$ & $<\mathbf{0 . 0 0 1} 1^{* *}$ \\
\hline $\begin{array}{l}\text { MPN group is used as reference for PN variable. } \\
\text { PN: Pronucleus number, CI: Confidence interval }\end{array}$ \\
\hline
\end{tabular}

Table 5. Generalized Linear Mixed Models for the effect of age on PN number

\begin{tabular}{|c|c|c|c|c|}
\hline & MPN & 2PN & MultiPN & \multirow{2}{*}{$\mathbf{F}, \mathbf{p}$} \\
\hline & n (\%) & n (\%) & n (\%) & \\
\hline \multicolumn{4}{|l|}{ Age } & $F=2.266$ \\
\hline$<35$ & 94 (10.5) & 744 (83.3) & $55(6.2)$ & $p=0.104$ \\
\hline$\geq 35$ & $52(13.4)$ & $320(82.7)$ & $15(3.9)$ & \\
\hline
\end{tabular}

developing from abnormal PN embryos were not transferred and all transfers were performed with blastocysts developing from 2PN embryos. Therefore, final IVF outcome between abnormal and 2PN transferred blastocysts were not evaluated. Chromosomal abnormalities are observed in $31.4 \%$ of the 3PN zygotes (17). Most of the embryos with multiPN are usually triploid and usually result in abortion (17). In our study, 42.8\% of multiPN embryos reached the blastocyst stage. Despite this high rate, the risk of abnormality may not be estimated without genetic screening before embryo transfer.

Development potentials of MPN zygotes with a wide pronuclear area or diameter are similar to those for $2 \mathrm{PN}$ zygotes (18). This may be observed due to early fusion of the male and female pronuclei. Embryos with MPN and two PB can be transferred only if a PGS diagnosis of euploidy is obtained and there is no other available normal fertilized embryo (19). The risk of chromosomal abnormalities in cells with MPN after ICSI is clearly higher than those after IVF and there are arguments that these embryos should never be transferred $(20,21)$.

A study of embryos with an abnormal pronucleus compared embryos with $0 \mathrm{PN}$ and MPN to embryos with $2 \mathrm{PN}$ on the fifth and sixth day of development and found no significant difference (22). In another similar study, implantation was not observed following transfer of the embryos developing from 0 PN and MPN (6). The embryos developing after MPN had the lowest rates of reaching blastocyst stage in our study. There is no consensus on the transfer of embryos with MPN in the literature, due to the limited number of studies.

An abnormal pronucleus may be iatrogenic, due to the methods used in assisted reproductive techniques. The incidence of multiPN is increased in cases of short incubation of gametes and early clearance of cumulus cells in oocytes (23). Embryos from MPN after IVF have higher rates of development on day 3, 5 and 6 compared to embryos from MPN after ICSI (24). Aneuploidy increases with maternal age, and abnormalities that occur after meiosis, such as mosaicism and polyploidy, may occur at a similar rate in all age groups (25). Kang et al. (26) reported that MPN and 3PN incidence increased in hyperstimulated cycles. It is controversial whether factors other than oocyte and sperm may also have an effect on abnormal pronuclei formation.

Different techniques and methods have been investigated for the correction of abnormally pronucleated embryos. One of these procedures is removing the excess pronucleus, by enucleation, from the zygotes with 3PN. A diploid embryo, with two pronuclei, was obtained after the procedure (27). The main limitation of this practice was the decision as to which pronucleus to remove. Removal of the wrong pronucleus may cause the embryo to carry two sets of maternal or paternal chromosomes. In a related study, one pronucleus of 
3PN zygotes was removed in the first group, and they were compared with a second group with 3PN zygotes followed without performing any procedure (28). The enucleation group had an increased potential for embryo formation, although the rate was still low when compared with $2 \mathrm{PN}$ zygotes (28). When karyotype analysis was performed on part of the enucleated group, $44.4 \%$ were identified as diploid and $55.5 \%$ as aneuploid.

Abnormal embryos can also be used to obtain human embryonic stem cells (hESC). hESC are cells with potential for self-renewal and differentiation into three germ layers (29). They can be used as a renewable source in cell transplantation for serious degenerative diseases. Huan et al. (30) obtained hESC from embryos with abnormal pronuclei, including 0 PN, MPN, and 3PN, and a normal pronucleus complement (2PN). All hESCs had normal chromosome content according to karyotype analysis. There was no structural difference among the obtained hESC cells, demonstrating distinct identity and karyotypic stability (30). Therefore, abnormal fertilized embryos can be used as a source for healthy hESC production.

However, genetic disorders can be observed in embryos developing after abnormal pronucleus formation. An embryo with mosaicism can correct itself by moving the mosaic cells towards the trophoblast during the development process, through the self-repairing mechanism of the cells (31). Tetraploid cells may be excluded from the primitive ectoderm lineage at an early stage. Another self-correcting mechanism is apoptosis and chromosomally abnormal embryos may undergo apoptosis (32).

A time-lapse system provides valuable morphokinetic data from embryo development in assisted reproduction techniques and each IVF laboratory should determine its own embryo selection criteria based on its own data (33). There is no consensus in the literature, whether abnormally fertilized embryos detected by time-lapse system are suitable for transfer.

Staessen et al. (5) reported the birth of two healthy children and one biochemical pregnancy after transfer of abnormally fertilized embryos. Grass and Trounson similarly recorded a healthy newborn in their study (34). One of the major successes was the birth of nine healthy infants from the transfer of embryos from MPN after IVF and implantation was observed in four of the embryos developing from MPN after ICSI (24). It is not right to declare that abnormally fertilized embryos should not be transferred. However, all abnormally fertilized embryos that have reached the blastocyst stage may not be eligible for transfer. In cases when there is no other embryo reaching the blastocyst stage, they can be transferred if no abnormality is detected after preimplantation genetic testing for aneuploidy (PGT-A).
Mutia et al. (35) analyzed 30 embryos developing from 3PN using next generation sequencing. They detected a normal chromosomal array in one third of them while the rest had abnormal chromosomes with the highest percentage of abnormality being triploidy (35). In vitro culture and chromosomal analysis of clinically discarded human embryos was investigated by Yao et al. (36). Blastocyst formation rates of 2PN embryos were higher than those for the abnormal 1PN, $3 \mathrm{PN}$, and $\geq 4 \mathrm{PN}$ embryos (36). Lim and Lee (37) reported three healthy live births, following PGT-A and transfer of four euploid, OPN-derived blastocysts to four patients. In addition, one on-going pregnancy was achieved by transfer of four euploid 1PN-derived blastocysts to four patients (37). In a similar study, Capalbo et al. (38) reported three live births by transfer of abnormally fertilized, oocyte-derived blastocysts after performing PGT-A. Hondo et al. (39) evaluated the rates of clinical pregnancy and live birth following transfer of frozenthawed 1PN- and 0PN-derived blastocysts. The pregnancy and live birth rates for $0 \mathrm{PN}$-derived embryos obtained by ICSI were similar to $2 \mathrm{PN}$-derived blastocysts. However, $1 \mathrm{PN}$-derived blastocysts had significantly lower rates (39).

\section{Study Limitation}

This study included some limitations. The status of the oocytes was only assessed on the first and fifth days. All data were analyzed from one center using a single protocol which was applied until day 5. Therefore, there is no data from day 6 onwards. In addition, if more extensive information from each day until the fifth day was available, there would have been a more detailed evaluation of the development of embryos after abnormal fertilization including factors such as pronucleus numbers, stages, and abnormalites in cell numbers until they reach the blastocyst stage. Scoring the embryos of MPN and multiPN origin reaching the blastocyst stage and comparing them with embryos of $2 \mathrm{PN}$ origin may further illuminate data on the quality of abnormally fertilized embryos.

\section{Conclusion}

Rate of blastocyst development after $2 \mathrm{PN}$ is significantly higher compared to those of the abnormal pronucleated embryos. MultiPN embryos have a higher chance to develop into blastocytes than MPN embryos. Large-scale research is difficult in this subject, because abnormal fertilization is not very common. Development of abnormally fertilized embryos usually stalls without reaching the fifth day. Owing to the low prevalence of abnormal fertilization, multicenter studies would delineate the effect of abnormal fertilization on embryonic development and provide more robust information about selection of abnormally pronucleated embryos for possible transfer. 
Ethics Committee Approval: The study protocol was approved by the Institutional Review Board of the Maltepe University Faculty of Medicine (approval number: 78, date: 22.09.2017).

\section{Informed Consent: Consent forms were obtained from the} patients.

Peer-review: Externally peer-reviewed.

Author Contributions: Surgical and Medical Practices: K.N.U., M.C., B.S.; Concept: M.C.; Design: M.C.; Data Collection or Processing: K.N.U., Ö.T.; Analysis or Interpretation: K.N.U., M.C., B.S., Ö.T.; Literature Search: K.N.U., M.C., E.U.; Writing: K.N.U., B.S., Ö.T.

Conflict of Interest: No conflict of interest is declared by the authors.

Financial Disclosure: The authors declared that this study received no financial support.

\section{References}

1. Azevedo AR, Pinho MJ, Silva J, Sá R, Thorsteinsdóttir S, Barros A, et al. Molecular cytogenetics of human single pronucleated zygotes. Reprod Sci 2014; 21: 1472-82.

2. Plachot M. Fertilization. Hum Reprod 2000; 15(Suppl 4): 19-30.

3. Kaufman MH. Early Mammalian Development: Parthenogenetic Studies. UK: Cambridge University Press; 1983.

4. Balakier H, Squire J, Casper RF. Characterization of abnormal one pronuclear human oocytes by morphology, cytogenetics and in-situ hybridization. Hum Reprod 1993; 8: 402-8.

5. Staessen C, Janssenwillen C, Devroey P, Steirteghem AC. Cytogenetic and morphological observations of single pronucleated human oocytes after in-vitro fertilization. Hum Reprod 1993; 8: 221-3.

6. Noyes N, Fino ME, Krey L, McCaffrey C, Adler A, Grifo J. Embryo biopsy: the fate of abnormal pronuclear embryos. Reprod BioMed Online 2008; 17: 782-8.

7. Prados FJ, Debrock S, Lemmen JG, Angerholm I. The cleavage stage embryo. In: ESHRE: Atlas of Human Embryology.

8. Flaherty SP, Payne D, Swann NJ, Matthews CD. Aetiology of failed and abnormal fertilization after intracytoplasmic sperm injection. Hum Reprod 1995; 10: 2623-9.

9. Flaherty SP, Payne D, Matthews CD. Fertilization failures and abnormal fertilization after intracytoplasmic sperm injection. Hum Reprod 1998; 13(Suppl 1): 155-64.

10. Rosenbusch B. The chromosomal constitution of embryos arising from monopronuclear oocytes in programmes of assisted reproduction. Reprod Med 2014; 2014: 418198.

11. Papale L, Fiorentino A, Montag M, Tomasi G. The zygote. Hum Reprod 2012; 27 (Suppl 1): i22-49.

12. Feenan K, Herbert M. Can "abnormally" fertilized zygotes give rise to viable embryos?. Hum Fertil (Camb) 2006; 9: 157-69.

13. Rosenbusch BE. A preliminary concept, deduced from cytogenetic analyses, for explaining different types of multipronuclear oocytes obtained after intracytoplasmic sperm injection. Fertil Steril 2010; 94: 2479-81.
14. Moore KL, Persaud TVN, Torchia MG. The Developing Human: Clinically Oriented Embryology. 9th. Philadelphia: Elsevier Saunders; 2013.

15. Chen Z, Yan J, Feng HL. Aneuploid analysis of tripronuclear zygotes derived from in vitro fertilization and intracytoplasmic sperm injection in humans. Fertil Steril 2005; 83: 1845-8.

16. Uyar E, Usal D, Selam B, Cincik M, Bagis T. IVF outcomes after hysteroscopic metroplasty in patients with T- shaped uterus. Fertil Res Pract 2019; 5: 15.

17. Rosenbusch B, Schneider M, Sterzik K. The chromosomal constitution of multipronuclear zygotes resulting from in-vitro fertilization. Hum Reprod 1997; 12: 2257-62.

18. Araki E, Itoi $F$, Honnma $H$, Asano $Y$, Oguri $H$, Nishikawa $K$. Correlation between the pronucleus size and the potential for human single pronucleus zygotes to develop into blastocysts: 1PN zygotes with large pronuclei can expect an embryo development to the blastocyst stage that is similar to the development of $2 \mathrm{PN}$ zygotes. J Assist Reprod Genet 2018; 35: 817-23.

19. Mateo S, Vidal F, Parriego M, Rodríguez I, Montalvo V, Veiga A, et al. Could monopronucleated ICSI zygotes be considered for transfer? Analysis through time-lapse monitoring and PGS. J Assist Reprod Genet 2017; 34: 905-11.

20. Greuner M, Montag M. Morphological Selection of Gametes and Embryos: 2PN/Zygote. In: Montag M, editor. A practical guide to selecting gametes and embryos. CRC press; 2014. p. 97-113.

21. Mateo S, Parriego M, Boada M, Vidal F, Coroleu B, Veiga A. In vitro development and chromosome constitution of embryos derived from monopronucleated zygotes after intracytoplasmic sperm injection. Fertil Steril 2013; 99: 897-902.

22. Yin BL, Hao HY, Zhang YN, Wei D, Zhang CL. Good quality blastocyst from non-/mono-pronuclear zygote may be used for transfer during IVF. Syst Biol Reprod Med 2016; 62: 139-45.

23. Zhou L, Wang J, Xiao L, Sun H, Wang Y, Geng L, et al. Differential effects of short co-incubation of gametes and early removal of cumulus cells in patients with different fertilizing capabilities. Reprod Biomed Online 2016; 32: 591-6.

24. Itoi F, Asano Y, Shimizu M, Honnma H, Murata Y. Birth of nine normal healthy babies following transfer of blastocysts derived from human single-pronucleate zygotes. J Assist Reprod Genet 2015; 32: 1401-7.

25. Munné S. Chromosome abnormalities and their relationship to morphology and development of human embryos. Reprod BioMed Online 2006; 12: 234-53.

26. Kang H, Neri QV, Wang A, Rosenwaks Z, Palermo GD. Withdrawal of FSH during controlled ovarian superovulation impairs fertilization with ICSI. Fertil Steril 2006; 86: S320.

27. Suss-Toby E, Gerecht Nir S, Amit M, Manor D, Itskoviz-Eldor J. Derivation of a diploid human embryonic stem cell line from a mononuclear zygote. Hum Reprod 2004; 19: 670-5.

28. Jin HX, Dai SJ, Song WY, Yao GD, Shi SL, Sun YP. Embryo developmental potential of microsurgically corrected human three-pronuclear zygotes. Syst Biol Reprod Med 2015; 61: 96-102.

29. Reubinoff BE, Pera MF, Fong CY, Trounson A, Bongso A. Embryonic stem cell lines from human blastocysts: somatic differentiation in vitro. Nat Biotechnol 2000; 18: 399-404.

30. Huan Q, Gao X, Wang Y, Shen Y, Ma W, Chen ZJ. Comparative evaluation of human embryonic stem cell lines derived from zygotes with normal and abnormal pronuclei. Dev Dyn 2010; 239: 425-38.

31. James RM, West JD. A chimaeric animal model for confined placental mosaicism. Hum Genet 1994; 93: 603-4.

32. Hardy K. Cell death in the mammalian blastocyst. Mol Hum Reprod 1997; 3: 919-25. 
33. Yalçınkaya E, Ergin EG, Calışkan E, Oztel Z, Ozay A, Ozörnek H Reproducibility of a time-lapse embryo selection model based on morphokinetic data in a sequential culture media setting. J Turk Ger Gynecol Assoc 2014; 15: 156-60.

34. Grass L, Trounson AO. Pregnancy and birth resulting from transfer of a blastocyst observed to have one pronucleus at the time of examination for fertilization. Hum Reprod 1999; 14: 1869-71.

35. Mutia K, Wiweko B, Iffanolida PA, Febri RR, Muna N, Riayati O, et al. The Frequency of Chromosomal Euploidy Among 3PN Embryos. J Reprod Infertil 2019; 20: 127-31.

36. Yao G, Xu J, Xin Z, Niu W, Shi S, Jin H, et al. Developmental potential of clinically discarded human embryos and associated chromosomal analysis. Sci Rep 2016; 6: 23995.
37. Lim AYX, Lee CSS. Embryos Arising from Apronuclear (OPN) and Unipronuclear (1PN) Have Similar Euploidy Rates with Those from 2PN and Should be Considered for Transfer. Fertil Reprod 2019; 1: 73-7.

38. Capalbo A, Treff N, Cimadomo D, Tao X, Ferrero S, Vaiarelli A, et al. bnormally fertilized oocytes can result in healthy live births: improved genetic technologies for preimplantation genetic testing can be used to rescue viable embryos in in vitro fertilization cycles. Fertil Steril 2017; 108: 1007-15.

39. Hondo S, Arichi A, Muramatsu H, Omura N, Ito $\mathrm{K}$, Komine $\mathrm{H}$, et al. Clinical outcomes of transfer of frozen and thawed single blastocysts derived from nonpronuclear and monopronuclear zygotes. Reprod Med Biol 2019; 18: 278-83. 